\title{
A novel role of copeptin as a biomarker for the prediction of treatment response in hyponatremia
}

\author{
Sang Heon Suh, Soo Wan Kim \\ Department of Internal Medicine, Chonnam National University Hospital, Chonnam National University Medical School, Gwangju, Republic \\ of Korea
}

\section{See Article on Page 371-382}

Arginine vasopressin (AVP) is a major regulating hormone in the body fluid homeostasis. Disorders of body fluid homeostasis may result in hyper- or hypoosmolar conditions. A typical example of hyper-osmolar disorders related to AVP is a diabetes insipidus, which is caused by impaired AVP synthesis (central diabetes insipidus) or impaired action in the kidney (nephrogenic diabetes insipidus). Hypoosmolar disorders include heterogeneous disease entities, such as syndrome of inappropriate antidiuresis (SIAD), and are largely related to dysregulated secretion of AVP. As disorders of body fluid homeostasis are closely related to AVP secretion imbalance, direct measurement of AVP to delineate the underlying pathophysiology has been tried. Indeed, plasma AVP levels substantially improve the diagnostic accuracy in a certain clinical context [1]. Nevertheless, the direct measurement of AVP has not been adopted in routine practice, mainly because of difficulties in specimen handling $[2,3]$.

Copeptin is secreted from the posterior pituitary as a precursor protein pre-provasopressin together with AVP and neurophysin II [4]. Copeptin is a peptide consisting of 39-amino acids, with a molecular weight of approximately $5 \mathrm{kDa}[4,5]$. Copeptin is very stable ex vivo, and easy to measure with sandwich immunoassay [5]. The main stimuli of the copeptin secretion are the same as those of AVPthe osmotic and other nonosmotic stimuli [6]. Although its physiological function is uncertain, the role of copeptin as a surrogate of AVP has been highlighted, as it is secreted in an equimolar amount to AVP. The use of copeptin is best illustrated in the differential diagnosis of polyuria-polydipsia syndrome (central and nephrogenic diabetes insipidus, and primary polydipsia), which has been suggested by Timper et al. [1]. Combined measurement of random and hypertonic saline-stimulated copeptin provides high diagnostic accuracy of $96 \%$ in the differential diagnosis of central diabetes insipidus from primary polydipsia, suggesting the diagnostic value of copeptin in hyperosmolar conditions [1]. On the other hand, the use of copeptin in the hypoosmolar disorder has been not been established until now.

In this regard, Go et al. [7] conducted analyses from a total of 100 participants in a prospective cohort to investigate the role of copeptin as a biomarker for the diagnosis and

Received: August 17, 2021; Accepted: August 17, 2021

Editor: Tae-Hyun Yoo, Yonsei University, Seoul, Republic of Korea

Correspondence: Soo Wan Kim

Department of Internal Medicine, Chonnam National University Medical School, 42 Jebongro, Gwangju 61469, Republic of Korea.

E-mail: skimw@chonnam.ac.kr

ORCID: https://orcid.org/0000-0002-3540-9004

Copyright (C) 2021 by The Korean Society of Nephrology

(a) This is an Open Access article distributed under the terms of the Creative Commons Attribution Non-Commercial and No Derivatives License (http:// creativecommons.org/licenses/by-nc-nd/4.0/) which permits unrestricted non-commercial use, distribution of the material without any modifications, and reproduction in any medium, provided the original works properly cited. 
prognosis in hypoosmolar disorders. All participants were defined as having symptomatic hyponatremia (corrected serum sodium of $\leq 125 \mathrm{mmol} / \mathrm{L}$ ) and were treated with hypertonic saline, where mean serum sodium and median copeptin levels were 117.9 and $16.9 \mathrm{pmol} / \mathrm{L}$, respectively. In the analyses to evaluate the copeptin levels as a marker for responses to hypertonic saline treatment in hyponatremic patients, low (below-median) copeptin levels at the baseline were associated with a significantly higher target correction rate in 6 hours (adjusted odds ratio [OR], 2.97; 95\% confidence interval [CI], 1.16-7.64; $\mathrm{p}=0.02$ ) and 24 hours (adjusted OR, 6.21; 95\% CI, 1.67-23.09; p = 0.006) after treatment start. Meanwhile, low copeptin levels at 24-hour after treatment was associated with a significantly higher overcorrection rate in 48-hour after treatment (adjusted OR, 18.00; 95\% CI, 1.59-203.45; $\mathrm{p}=0.02$ ). The authors presented a plausible explanation for these findings that low baseline copeptin may indicate less chance of previous hyperosmolar or hypovolemic stimuli, thereby enhancing the response to a hypertonic saline infusion (Table 1). The authors also suggested that a low copeptin level at 24 hours after treatment may indicate that a greater excretion of free water can occur to augment the effect on serum sodium level by the same amount of hypertonic saline (Table 1).

In the current study, Go et al. [7] also investigated the usefulness of copeptin for differentiating etiologies of hyponatremia. The participants were classified into five categories, based on the patient's history, physical examination, and laboratory test results: (1) decreased extracellular fluid (ECF) volume due to renal Na loss (e.g., diuretics, especially thiazides); (2) decreased ECF volume due to nonrenal sodium loss (e.g., gastrointestinal Na loss or third spacing: vomiting, diarrhea, or malnutrition); (3) increased ECF volume (e.g., heart failure, liver cirrhosis, and nephrotic syndrome); (4) normal ECF volume with adrenal insufficiency; and (5) normal ECF volume fulfilling the essential criteria for SIAD. Although the ratio of copeptin-to-urine sodium improved the differential diagnosis of hyponatremic patients with insufficient effective circulatory volume from the others, the authors were not able to present any cutoff values of copeptin levels to discriminate the etiologies of hyponatremia, due to widely overlapping copeptin levels among the different etiologies of hyponatremia and large variability within a single category. This finding is in agreement with a previous report [8] and seems clear that hyponatremia is not solely dependent on inappropriate AVP secretion, but also other factors, such as medications, volume status, or stress [9].

The cost-effectiveness of copeptin measurement in hyponatremic patients is a remaining question, which should be considered for routine practice. As it is still important to repeatedly measure serum sodium level in the treatment of hyponatremic patients, copeptin should be measured in addition to serum sodium measurement. Further, although osmotic demyelination syndrome is obviously a dreaded complication of overcorrection in the treatment of hyponatremia, its frequency is rare [10]. Hence, it should be validated whether the cost of routine copeptin measurement could be balanced by the prevention of osmotic demyelination syndrome.

In summary, Go et al. [7] suggested a novel role of copeptin as a biomarker for the prediction of treatment response in hyponatremia. The authors demonstrated that low copeptin levels at the baseline are associated with a significantly higher target correction rate in 6 and 24 hours after hypertonic saline treatment start, and that low copeptin levels at 24-hour after hypertonic saline treatment is associated with a significantly higher overcorrection rate in the next 24 hours. The cost-effectiveness of copeptin measurement in hyponatremic patients is a remaining question requiring further consideration.

Table 1. Interpretation of plasma copeptin level during hypertonic saline treatment of hyponatremic patients, suggested by Go et al. [7]

\begin{tabular}{lcc}
\hline Plasma copeptin & Low & High \\
\hline Putative status of plasma AVP & Low & High \\
Interpretation & $\begin{array}{c}\text { B } \\
\text { haseline: Less chance of previous hyperosmolar or } \\
\text { hypovolemic stimuli } \rightarrow \text { Enhanced response to a } \\
\text { hypertonic saline infusion }\end{array}$ & $\begin{array}{c}\text { B Baseline: More chance of previous hyperosmolar } \\
\text { or hypovolemic stimuli } \rightarrow \text { Poor response to a } \\
\text { hypertonic saline infusion }\end{array}$ \\
$\begin{array}{c}\text { 24-hour after hypertonic saline treatment: Greater } \\
\text { excretion of free water } \rightarrow \text { Increased risk of overcor- } \\
\text { rection by the same amount of hypertonic saline }\end{array}$ & $\begin{array}{c}\text { 24-hour after hypertonic saline treatment: Less ex- } \\
\text { cretion of free water } \rightarrow \text { Decreased risk of overcor- } \\
\text { rection by the same amount of hypertonic saline }\end{array}$ \\
\hline
\end{tabular}

Note that low and high copeptin levels indicate below and above median copeptin level (16.9 pmol/L), respectively.

AVP, arginine vasopressin. 


\section{Conflicts of interest}

All authors have no conflicts of interest to declare.

\section{ORCID}

Sang Heon Suh, https://orcid.org/0000-0003-3076-3466

Soo Wan Kim, https://orcid.org/0000-0002-3540-9004

\section{References}

1. Timper K, Fenske W, Kühn F, et al. Diagnostic accuracy of copeptin in the differential diagnosis of the polyuria-polydipsia syndrome: a prospective multicenter study. J Clin Endocrinol Metab 2015;100:2268-74.

2. Refardt J, Winzeler B, Christ-Crain M. Copeptin and its role in the diagnosis of diabetes insipidus and the syndrome of inappropriate antidiuresis. Clin Endocrinol (Oxf) 2019;91:22-32.

3. Kluge M, Riedl S, Erhart-Hofmann B, Hartmann J, Waldhauser F. Improved extraction procedure and RIA for determination of arginine8-vasopressin in plasma: role of premeasurement sample treatment and reference values in children. Clin Chem 1999;45:98-103.

4. Land H, Schütz G, Schmale H, Richter D. Nucleotide sequence of cloned cDNA encoding bovine arginine vasopressin-neuro- physin II precursor. Nature 1982;295:299-303.

5. Morgenthaler NG, Struck J, Alonso C, Bergmann A. Assay for the measurement of copeptin, a stable peptide derived from the precursor of vasopressin. Clin Chem 2006;52:112-9.

6. Christ-Crain M, Fenske W. Copeptin in the diagnosis of vasopressin-dependent disorders of fluid homeostasis. Nat Rev Endocrinol 2016;12:168-76.

7. Go S, Kim S, Son HE, et al. Association between copeptin levels and treatment responses to hypertonic saline infusion in patients with symptomatic hyponatremia: a prospective cohort study. Kidney Res Clin Pract 2021 Jul 8 [Epub]. DOI: 10.23876/ j.krcp.20.233.

8. Nigro N, Winzeler B, Suter-Widmer I, et al. Evaluation of copeptin and commonly used laboratory parameters for the differential diagnosis of profound hyponatraemia in hospitalized patients: 'The Co-MED Study'. Clin Endocrinol (Oxf) 2017;86:456-62.

9.Fiordoliva I, Meletani T, Baleani MG, et al. Managing hyponatremia in lung cancer: latest evidence and clinical implications. Ther Adv Med Oncol 2017;9:711-9.

10. Baek SH, Jo YH, Ahn S, et al. Risk of overcorrection in rapid intermittent bolus vs slow continuous infusion therapies of hypertonic saline for patients with symptomatic hyponatremia: the SALSA Randomized Clinical Trial. JAMA Intern Med 2021;181:81-92. 